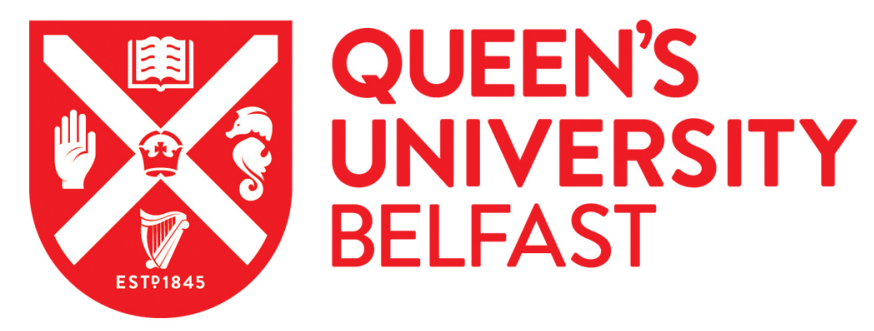

\title{
Molecular confirmation of the unique phenotype of epidermolysis bullosa simplex with mottled pigmentation
}

Irvine, A. D., Rugg, E. L., Lane, E. B., Hoare, S., Peret, C., Hughes, A., \& Heagerty, A. H. (2001). Molecular confirmation of the unique phenotype of epidermolysis bullosa simplex with mottled pigmentation. British Journal of Dermatology, 144(1), 40-45.

Published in:

British Journal of Dermatology

Queen's University Belfast - Research Portal:

Link to publication record in Queen's University Belfast Research Portal

\section{General rights}

Copyright for the publications made accessible via the Queen's University Belfast Research Portal is retained by the author(s) and / or other copyright owners and it is a condition of accessing these publications that users recognise and abide by the legal requirements associated with these rights.

Take down policy

The Research Portal is Queen's institutional repository that provides access to Queen's research output. Every effort has been made to ensure that content in the Research Portal does not infringe any person's rights, or applicable UK laws. If you discover content in the Research Portal that you believe breaches copyright or violates any law, please contact openaccess@qub.ac.uk. 


\title{
Molecular confirmation of the unique phenotype of epidermolysis bullosa simplex with mottled pigmentation
}

\author{
A.D.IRVINE, ${ }^{*} \dagger \dagger$ E.L.RUGG, $\dagger$ E.B.LANE, + S.HOARE, $§$ C.PERET, $\uparrow$ A.E.HUGHES $\dagger \dagger$ AND \\ A.H.HEAGERTY** \\ *Department of Dermatology, The Hospital for Children, Great Ormond Street, London WC1N 3JH, U.K. \\ $\dagger$ Centre for Cutaneous Research, St Bartholomew's and the Royal London School of Medicine and Dentistry, \\ Queen Mary and Westfield College, 2 Newark Street, London E1 2AT, U.K. \\ $\ddagger$ Department of Anatomy and Physiology, The University of Dundee, MSI/WTB Complex, Dow Street, Dundee DD1 5EH, U.K. \\ §Department of Oncology, Glasgow United Hospitals, Glasgow, U.K. \\ qDepartment of Obstetrics and Gynaecology, Royal Free Hospital, London, U.K. \\ t†Department of Medical Genetics, The Queen's University Belfast, A Floor, Belfast City Hospital, Belfast BT9 7AB, U.K. \\ **Department of Dermatology, Heartlands and Solihull NHS Trust, Lode Lane, West Midlands B91, U.K. \\ Accepted for publication 20 July 2000
}

Summary

Epidermolysis bullosa simplex with mottled pigmentation (MIM 131960; EBS-MP) is a subtype of epidermolysis bullosa simplex first reported in $1979 .{ }^{1}$ Since this initial description, seven further families have been reported. ${ }^{2-6}$ Acral blistering with onset in early childhood is a constant feature and, as with the Weber-Cockayne EBS (EBS-WC) subtype there is often marked seasonal variance in severity. Bullae, which are often blood-filled, occur most commonly on the limbs and heal spontaneously without milia formation. ${ }^{1-3}$ Histologically, blistering in EBS-MP resembles that found in EBS-WC and EBS-Koebner. ${ }^{1,7}$

Correspondence: Dr A.D.Irvine. E-mail: irvo67@hotmail.com
The mottled pigmentation has a very distinctive appearance and shows predominance for the lower abdomen, axillae and limbs. Discrete pigmented macules $2-5 \mathrm{~mm}$ in diameter, usually appear in infancy and remain present throughout life. Histology shows increased melanin content in dermal melanophages. ${ }^{1}$ Ultrastructural analysis of pigmented areas demonstrates abundant mature melanosomes within basal keratinocytes. ${ }^{7,8}$ Focal or punctate keratoderma of the palms and soles is a frequent finding. Palmoplantar keratoderma presents much later in life than blistering and pigmentary changes. Dystrophic, thickened nails are commonly reported. ${ }^{1,4}$ 


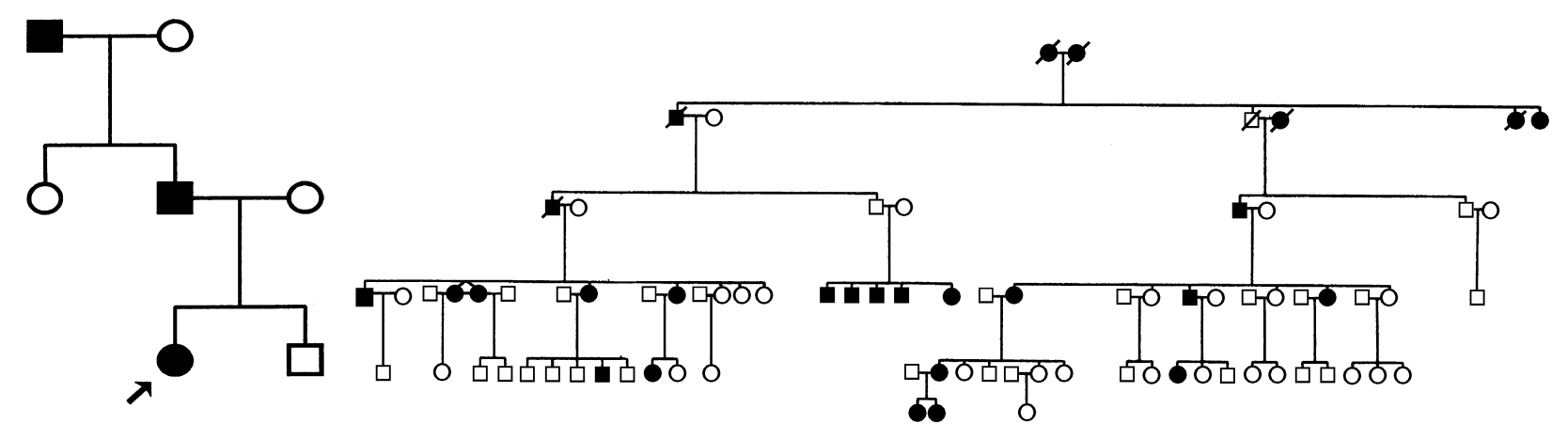

Family 1

Figure 1. Family pedigrees.

The genetic basis of EBS-MP has now been ascribed in five independent kindreds to a heterozygous point mutation $(465 \mathrm{C} \rightarrow \mathrm{T}$; P25L), in the non-helical V1 domain of $\mathrm{K} 5 .^{5-7}$ Here we report a clinical, ultrastructural and molecular study of two British families, which were among the earliest families to be clinically characterized as EBS-MP. ${ }^{2}$ The P25L mutation was identified in all affected members of each of these families, bringing the total number of EBS-MP families with this mutation to seven. This recurrent $\mathrm{C} \rightarrow \mathrm{T}$ transition may uniquely cause the EBS-MP phenotype and suggests additional roles for keratin intermediate filaments (IF).

\section{Patients and methods}

\section{Subjects}

Following local ethical committee approval, two British families originally reported in $1981^{2}$ were traced (Fig. 1) and individually examined by the authors (ADI/AH). Biopsy material was taken for examination by electron microscopy.

\section{Electron microscopy}

Specimens were fixed in 2\% glutaraldehyde in Sörensen's buffer and then $2 \%$ osmium tetroxide using a standard protocol. One micron semithin sections were cut on an Ultracut E (Reichert-Jung) and stained with Toluidine Blue. Tissue was embedded in Araldite resin (Araldite resin embedding kit-E009, Taab Laboratories Equipment Ltd, Aldermaston, U.K.). Electron microscopy was performed on a Philips EM410.

\section{Molecular analysis}

Genomic DNA extracted from peripheral leucocytes was used as a template for polymerase chain reaction (PCR) amplification. A $546 \mathrm{bp}$ fragment of KRT5 was amplified in a $50-\mu \mathrm{L}$ reaction as previously described. ${ }^{5}$ PCR products were sequenced in both directions on an ABI Prism 377 sequencer with AmpliTaq ${ }^{\circledR}$ DNA polymerase and dye-labelled terminators in accordance with Perkin Elmer protocol P/N 402078. As the P25L mutation neither created nor destroyed a restriction site, the mutation was excluded from unaffected family members and 100 unaffected, unrelated controls using PCR amplification of specific alleles (PASA). A $140 \mathrm{bp}$ fragment was amplified using a common anti-sense primer and either a wild-type or mutant sense primer as previously described. ${ }^{5}$ PCR products were resolved by agarose gel electrophoresis.

\section{Results}

\section{Clinical findings}

Two affected family members were examined in family 1. The affected father (II.2) and the proband (III.1) both had a history of lifelong blistering with seasonal exacerbation in the summer months and diminution of severity with increasing age. Both individuals had characteristic mottled pigmentation distributed over the trunk and distal extensor aspects of the limbs. The father had marked palmoplantar keratoderma, which developed at age 16 years but this was not yet a feature in his 19-year-old daughter. Similar clinical features 

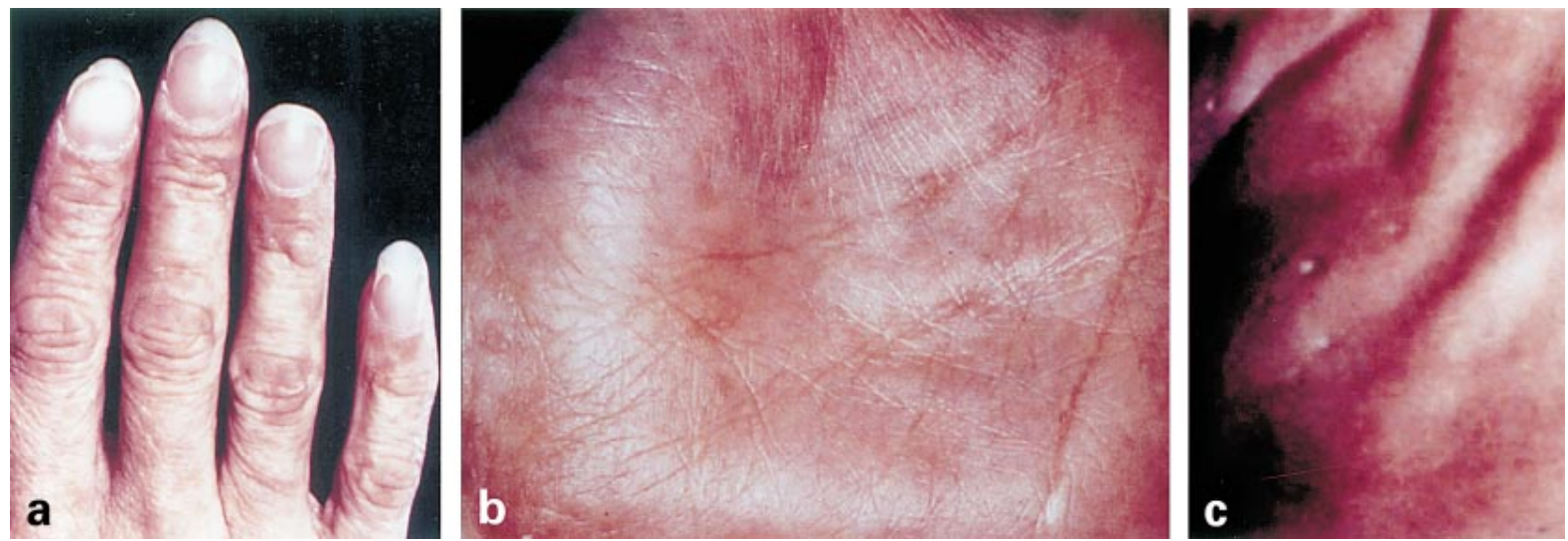

Figure 2. Clinical features. Three to $5 \mathrm{~mm}$ pigmented macules are seen over the dorsum (a) and palm (b) of the hand. (c) Detail of the punctate plantar keratoderma.

were noted in family 2. Discrete pigmented macules of 2-5 $\mathrm{mm}$ were observed over the trunk, limbs and hands (Fig. 2a,b). Punctate or focal palmoplantar keratoderma developed in most family members in late teenage years (Fig. 2c).

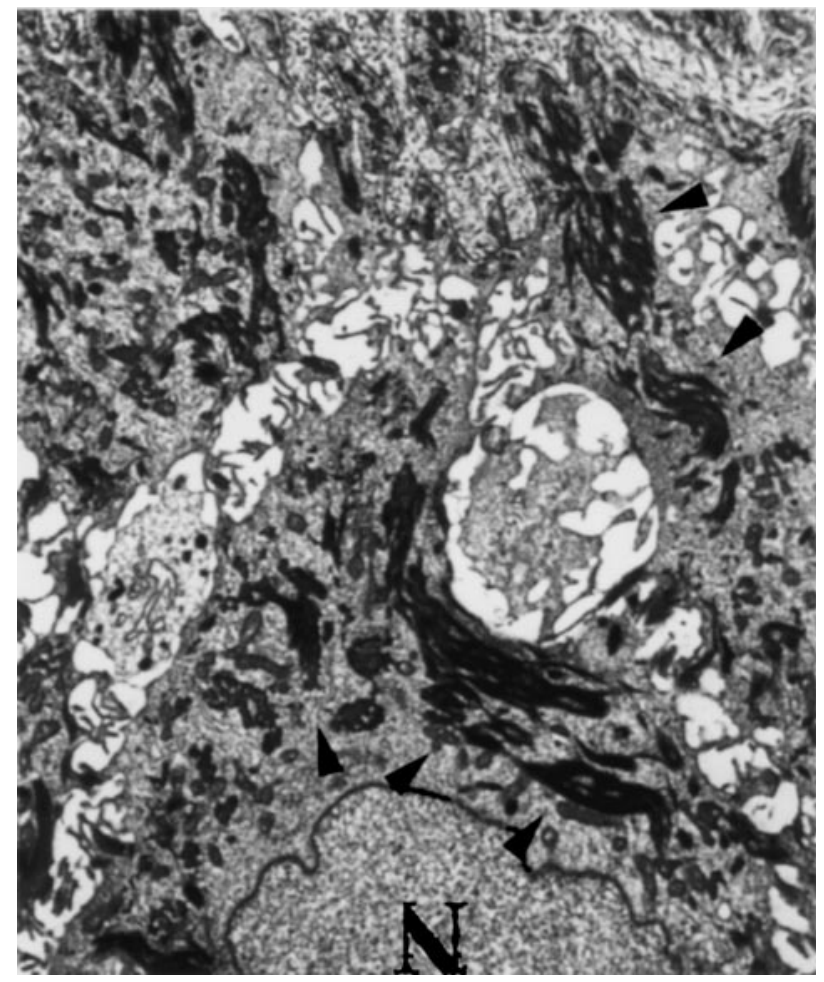

Figure 3. Basal cell cytolysis and keratin filament disorganization. Transmission electron microscopy of the basal layer in an affected individual. The level of splitting and basal cell degeneration is similar to that seen in other epidermolysis bullosa simplex subtypes. Areas of vacuolization are seen adjacent to the nucleus. Atypical clumped keratin intermediate filaments are seen (black arrowheads). N, nucleus (original magnification $\times 15,000$ ).

\section{Electron microscopy}

The basal cell layer level of splitting and basal layer keratinocyte degeneration noted was similar to that seen in other EBS subtypes. Areas of vacuolization were seen adjacent to the nucleus, atypical clumped keratin

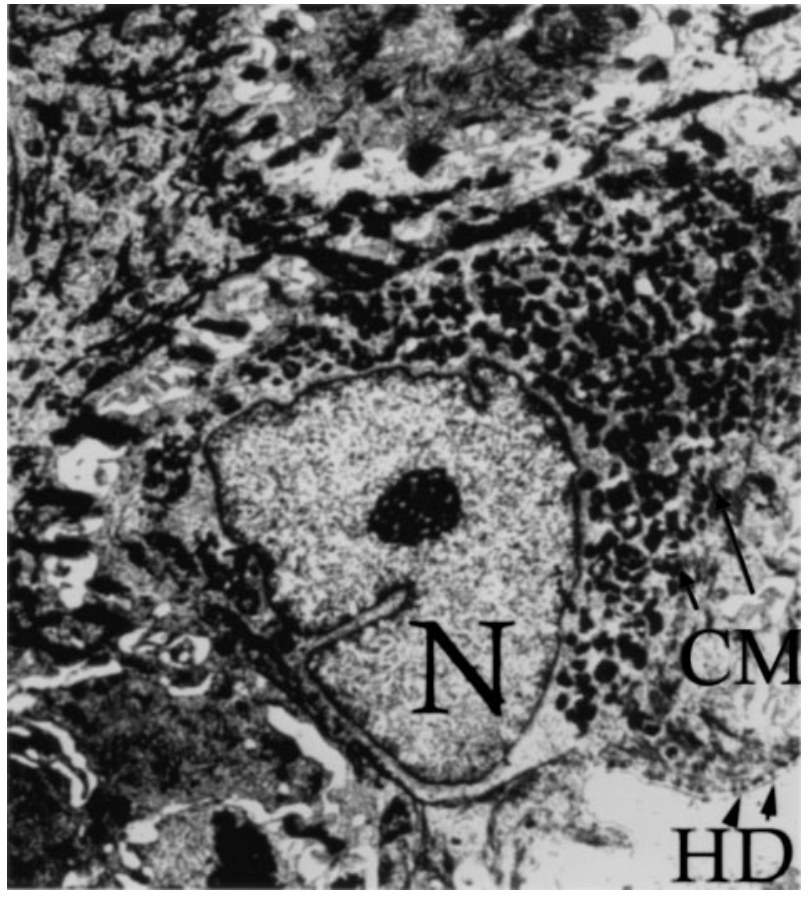

Figure 4. Ultrastructure of pigmentation in epidermolysis bullosa simplex, with the additional feature of mottled pigmentation. Transmission electron micrograph of a representative area of pigmentation from an affected individual in family 2 . The most striking feature is the aggregation of multiple densely packed compound melanosomes in the perinuclear cytoplasm of a basal keratinocyte. N, nucleus; $\mathrm{CM}$, compound melanosome; HD, hemidesmosome (original magnification $\times 15,000$ ). 

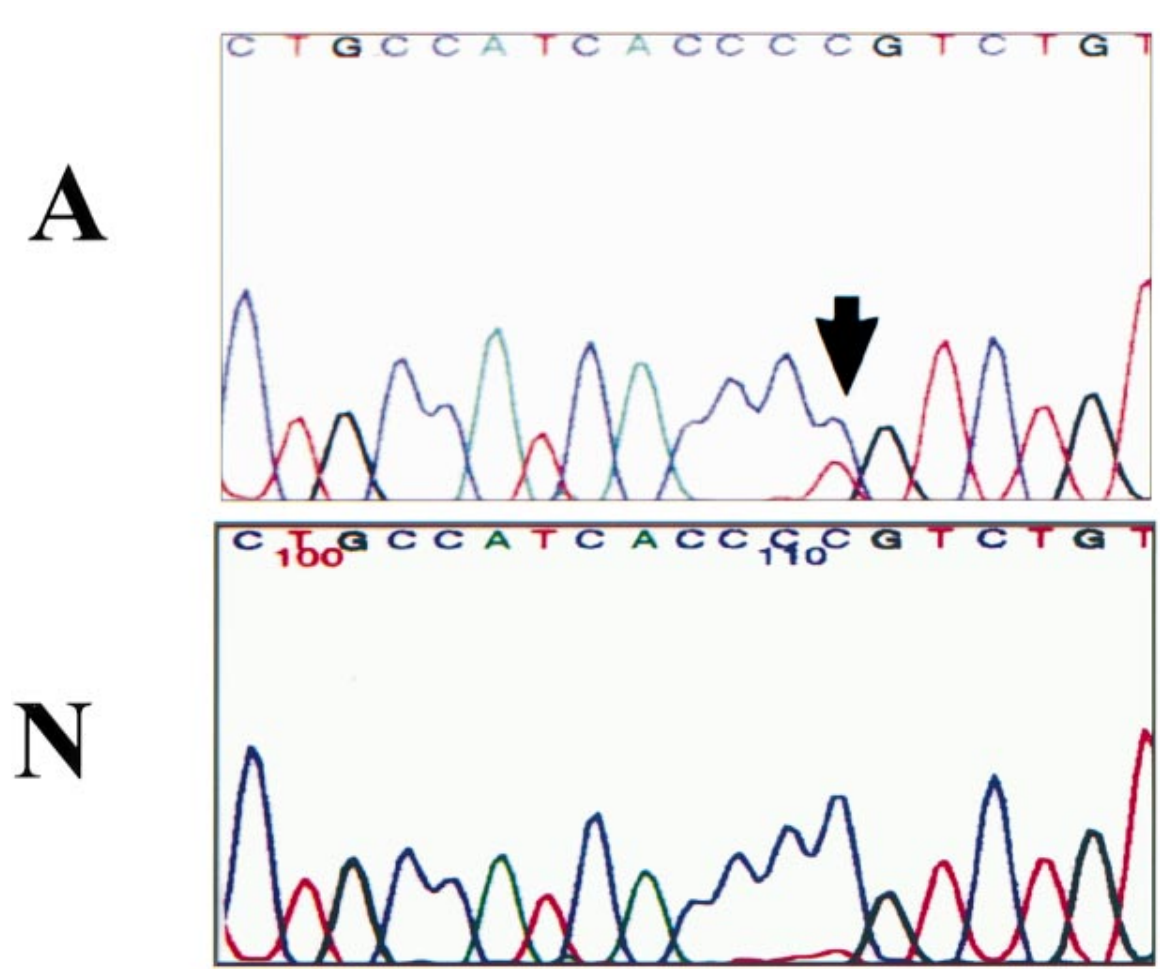

Figure 5. Automated sequence data. A heterozygous $\mathrm{C} \rightarrow \mathrm{T}$ transition is demonstrated at base position 465 , predicting the amino acid substitution P25L. A, affected individual; N, normal control.
IFs were also demonstrated (Fig. 3). Examination of a representative area of pigmentation from an affected individual showed dense packing of compound melanosomes around the keratinocyte nucleus (Fig. 4).

\section{Molecular analysis}

Direct sequencing of the KRT5 gene in all affected individuals revealed a heterozygous $\mathrm{C} \rightarrow \mathrm{T}$ transition at base position 465 (Fig. 5; GenBank Accession number M21389), within the V1domain of K5, predicting a proline-to-leucine substitution at codon 25. This substitution was confirmed in these families and excluded from 100 unaffected unrelated individuals using PASA as previously described. ${ }^{5}$ The P25L mutation occurs within an 18 amino acid motif, which is highly conserved in a number of IFs across several species, within this motif a particular SxxSxxxPxxxR pattern is especially conserved (Fig. 6).

\section{Discussion}

A proline-to-leucine substitution was identified at position 25 of the amino-terminal head domain of K5

\begin{tabular}{|c|c|c|c|}
\hline uman K5 & F $\mathbf{S}$ T A S A & T S & 9( \\
\hline un & $S$ A S S A R L & G V & $8(1)$ \\
\hline 6 & A R V $\mathbf{P}$ & & (1) \\
\hline e II keratin & A C G $\mid \mathbf{P}$ & $\mathrm{R}$ & (2) \\
\hline & L $R \mid P$ & & (2) \\
\hline & L R $\mid \mathbf{P}$ & & $5(2)$ \\
\hline & A L $R \mid \mathbf{P}$ & S R S L Y S S & $2(2)$ \\
\hline licken lamin $\mathrm{A}$ & $\begin{array}{lllll}\text { A } & S & S & R & R\end{array}$ & $\mathrm{~L} S$ A S S & $\mathrm{X} 16879(1)$ \\
\hline
\end{tabular}

Figure 6. Conservation of an 18 amino acid motif within the V1 domain of $\mathrm{K} 5$ and related regions of different classes of intermediate filaments. There is considerable conservation of this motif across several species and proteins. The substituted proline is boxed. An SxxSxxxPxxR pattern is conserved in $100 \%$ of these proteins. Numbers in brackets refer to accession numbers in the GenBank (1) and SWISS-PROT (2) databases. 
in two unrelated families with EBS-MP. This identical mutation has been reported in five other unrelated families, one from Germany, one from the U.S.A., one from Northern Ireland and two from the Netherlands. ${ }^{5-7}$ Two of these cases were sporadic patients, ${ }^{5,6}$ making a common founder effect an unlikely explanation for this recurrent mutation. This mutation has been previously reported as P24L, based on the numbering suggested by Lersch et al., ${ }^{9}$ which ignores the initial methionine; currently all other KRT5 mutations are numbered according to GenBank M21389, which includes methionine. For consistency with other reported keratin mutations we have used GenBank M21389 to identify the mutation as P25L. We suggest that this designation is used in future reports.

The ultrastructural appearances shown here (Figs 3 and 4) of clumped keratin filaments in the basal layer of the epidermis suggest that the P25L mutation has an effect on filament structure and integrity. In vitro studies on the P25L mutation have shown that the mutant protein is able to form filaments but have suggested that there are some effects on the length and uniformity of filaments. ${ }^{7}$ As the P25L mutation lies outside the central helical rod domain, the means by which it exerts a dominant-negative effect on the cytoskeleton with subsequent epidermolysis is almost certainly different from those implicated in other EBS subtypes. Currently, this mechanism is to some degree a matter of speculation, informed by examining functional data from keratins and other IF proteins. In vitro assembly studies have shown that the amino-terminal of $\mathrm{K} 5$ is the most important of the four head/tail domains involved in $\mathrm{K} 5 / 14$ heterodimer and higherorder IF assembly, with particular effects on filament elongation and lateral alignment. ${ }^{10}$ The importance of these interactions may further be inferred from in vitro assembly studies in other IFs. There is, for example, compelling evidence of a crucial part for the aminoterminal domain in higher IF assembly from in vitro studies on the type III IF vimentin. This IF has a highly conserved nine-peptide motif within the V1 domain, which is critical for co-ordinating intratetrameric reactions and protofilament formation ${ }^{11}$ and a single missense mutation within this motif can seriously derange IF assembly. ${ }^{12}$ Other in vitro studies have demonstrated a role for the vimentin head domain in correct alignment of the two coiled-coil structure and in stabilization of protofilaments. ${ }^{13}$

The P25L substitution occurs within an 18 amino acid motif within the V1 domain that is highly conserved across many IFs, including vimentin, K5 and all K6 isoforms (Fig. 6), suggesting an important function for this site. The most conserved component within this motif is an SxxSxxxPxxxR sequence. Conservation of proline and arginine residues near serine or threonine residues is characteristic of a phosphorylation site and disruption of this potential phosphorylation site is one molecular mechanism whereby the P25L substitution could cause impairment of cytoskeletal function. If this hypothesis is correct then presumably mutations in any of the other conserved residues in this motif (i.e. S18, S21 and R29) should also cause EBS-MP. The predominance of mutations at residue 25 may be due to a $\mathrm{CpG}$ site within this codon causing increased susceptibility to $\mathrm{C} \rightarrow \mathrm{T}$ transitions. The sequence PSVS in $\mathrm{K} 5$ is also a putative O-linked single $\mathrm{N}$-acetylglucosamine glycosylation motif, ${ }^{14}$ suggesting an alternative molecular pathogenic mechanism. A number of IF proteins contain this motif and this site has been shown to be glycosylated in keratin 18 and neurofilament proteins L and M. While the significance of this post-translational modification remains unclear, glycosylation of IF proteins appears to be restricted to their head and tail domains and it has been speculated that these modifications are involved in filament assembly and/ or protein-IF interactions. ${ }^{14,15}$

One of the most intriguing aspects of EBS-MP is the aetiology of pigmentation. The evidence strongly suggests that the P25L mutation underlies both epidermolysis and the associated pigmentation, although the exact molecular mechanism remains unclear. The ultrastructural appearances of EBS-MP could be interpreted as consistent with postinflammatory pigmentation, although this fails to explain why MP is not seen in EBS subtypes. The mutation lies within a region of $\mathrm{K} 5$ that protrudes along the filament surface, ${ }^{16}$ allowing for an alternative hypothesis where the P25L mutation causes pigmentation by altering keratin filament architecture in a way that influences melanin granule aggregation in basal cells. The mechanism underlying this disruption could be disruption of phosphorylation or glycosylation. Further in vitro mutagenesis studies with molecular models of the keratin 5 amino-terminal head domain will be necessary in order to elucidate the role of this domain fully in keratin interactions with other structural molecules and intracellular organelles. Punctate or focal keratoderma is a common manifestation in adult EBS-MP patients and again, the molecular basis of this is speculative. While a diverse range of molecular 
defects, including dominant-negative mutations in several keratins ${ }^{17}$ and haploinsufficiency of keratinassociated molecules such as desmoplakin ${ }^{18}$ as well as dominant negative mutations/haploinsufficiency of desmosomal cadherins ${ }^{19}$ are known to cause keratoderma, the exact final molecular basis of hypercornification remains unclear in all keratodermas. Cell cytolysis leading to disruption of cell-cell signalling and the subsequent disruption to the control of terminal differentiation may represent a 'common final pathway.' Desmoplakin binding sites have been localized to the first 71 amino acids of the aminoterminal domain of $\mathrm{K} 5,{ }^{20}$ so the $\mathrm{P} 25 \mathrm{~L}$ substitution could potentially cause palmoplantar keratoderma either directly by disrupting keratin filaments or indirectly by disrupting binding sites within the V1 domain for DPI or other IF-related proteins.

\section{Acknowledgments}

ADI was supported by a British Society for Investigative Dermatology Young Investigator Fellowship. We are grateful to Dr R.D.Peachey for assistance in tracing family 1.

\section{References}

1 Fischer T, Gedde-Dahl T Jr. Epidermolysis bullosa simplex and mottled pigmentation: a new dominant syndrome. I. Clinical and histological features. Clin Genet 1979; 15: 228-38.

2 Boss JM, Matthews CN, Peachey RD et al. Speckled hyperpigmentation, palmoplantar punctate keratoses and childhood blistering: a clinical triad, with variable associations. A report of two families. Br J Dermatol 1981; 105: 579-85.

3 Coleman R, Harper JI, Lake BD. Epidermolysis bullosa simplex with mottled pigmentation. Br J Dermatol 1993; 128: 679-85.

4 Combemale P, Kanitakis J. Epidermolysis bullosa simplex with mottled pigmentation. Case report and review of the literature. Dermatol 1994; 189: 173-8.

5 Irvine AD, McKenna KE, Jenkinson $\mathrm{H}$ et al. A mutation in the V1 domain of keratin 5 causes epidermolysis bullosa simplex with mottled pigmentation. J Invest Dermatol 1997; 108: 809-10.

6 Moog U, de Die-Smulders CE, Scheffer H et al. Epidermolysis bullosa simplex with mottled pigmentation: clinical aspects and confirmation of the P24L mutation in the KRT5 gene in further patients. Am J Med Genet 1999; 86: 376-9.

7 Uttam J, Hutton E, Coulombe PA et al. The genetic basis of epidermolysis bullosa simplex with mottled pigmentation. Proc Natl Acad Sci USA 1996; 93: 9079-84.

8 Gedde-Dahl T, Anton-Lamprecht I, Fischer T. Ultrastructural features of a new syndrome: Epidermolysis bullosa and mottled pigmentation. J Cutan Pathol 1981; 8: 161(Abstr.).

9 Lersch R, Stellmach V, Stocks C et al. Isolation, sequence, and expression of a human keratin $\mathrm{K} 5$ gene: transcriptional regulation of keratins and insights into pairwise control. Mol Cell Biol 1989; 9: 3685-97.

10 Wilson AK, Coulombe PA, Fuchs E. The roles of K5 and K14 head, tail, and R/K L L E G E domains in keratin filament assembly in vitro. J Cell Biol 1992; 119: 401-14.

11 Hofmann I, Herrmann H. Interference in vimentin assembly in vitro by synthetic peptides derived from the vimentin head domain. J Cell Sci 1992; 101: 687-700.

12 Herrmann H, Hofmann I, Franke WW. Identification of a nonapeptide motif in the vimentin head domain involved in intermediate filament assembly. J Mol Biol 1992; 223: 637-50.

13 Traub P, Scherbarth A, Wiegers W et al. Salt-stable interaction of the amino-terminal head region of vimentin with the alphahelical rod domain of cytoplasmic intermediate filament proteins and its relevance to protofilament structure and filament formation and stability. J Cell Sci 1992; 101: 363-81.

$14 \mathrm{Ku}$ NO, Omary MB. Identification and mutational analysis of the glycosylation sites of human keratin 18. J Biol Chem 1995; 270: $11820-7$.

15 Dong DL, Xu ZS, Chevrier MR et al. Glycosylation of mammalian neurofilaments. Localization of multiple O-linked N-acetylglucosamine moieties on neurofilament polypeptides L and M. J Biol Chem 1993; 268: 16679-87.

16 Steinert PM, Rice RH, Roop DR et al. Complete amino acid sequence of a mouse epidermal keratin subunit and implications for the structure of intermediate filaments. Nature 1983; 302: 794-800.

17 Irvine AD, McLean WH. Human keratin diseases: the increasing spectrum of disease and subtlety of the phenotype-genotype correlation. Br J Dermatol 1999; 140: 815-28.

18 Armstrong DK, McKenna KE, Purkis PE et al. Haploinsufficiency of desmoplakin causes a striate subtype of palmoplantar keratoderma. Hum Mol Genet 1999; 8: 143-8.

19 Rickman L, Simrak D, Stevens HP et al. N-terminal deletion in a desmosomal cadherin causes the autosomal dominant skin disease striate palmoplantar keratoderma. Hum Mol Genet 1999; 8: 971-6.

20 Kouklis PD, Hutton E, Fuchs E. Making a connection: direct binding between keratin intermediate filaments and desmosomal proteins. J Cell Biol 1994; 127: 1049-60. 